\title{
DISCRETE CELLS PROPERTIES \\ IN THE BOUNDARY SET SETTING
}

\author{
PHILIP L. BOWERS
}

\begin{abstract}
Let $X$ be the complement of a $\sigma$-Z-set in a locally compact separable ANR. It is proved that $X$ satisfies the discrete $n$-cells property for each nonnegative integer $n$ if and only if $X$ satisfies the discrete approximation property. As a consequence, Hilbert space manifolds that arise as complements of boundary sets in Hilbert cube manifolds are characterized in terms of their homological structure coupled with a minimal amount of general positioning.
\end{abstract}

1. Introduction. H. Torunczyk characterizes those infinite-dimensional manifolds modeled on the Hilbert cube and Hilbert space entirely in terms of geometric general position properties. Hilbert cube manifolds are precisely those locally compact ANR's $X$ for which arbitrary pairs of maps of the Hilbert cube into $X$ are approximable by maps whose images are disjoint [To1]. This general position property easily is seen to be equivalent to the disjoint $n$-cells property (maps of the $n$-cell into $X$ are approximable by maps with disjoint images) holding simultaneously for all nonnegative integers $n$. Hilbert space manifolds are precisely those topologically complete separable ANR's $X$ for which arbitrary sequences of maps of the Hilbert cube into $X$ are strongly approximable by maps whose images form a discrete family [To2, BBMW]. This latter general position property is known as the discrete approximation property, and the question naturally arises as to whether or not this property is equivalent to the analogous $n$-dimensional properties holding simultaneously for all nonnegative integers $n$.

Recently, M. Bestvina, J. Mogilski, J. Walsh, and the author constructed examples of topologically complete separable ANR's that satisfy the discrete $n$-cells property for each nonnegative integer $n$, but fail to satisfy the discrete approximation property [BBMW]. (Precise definitions of the aforementioned terms are found in \$2.) None of the examples constructed in [BBMW] arise as complements of $\sigma$-Z-sets in locally compact separable ANR's, a common source of Hilbert space manifolds. Our Main Result appears in $\$ 3$ and shows that no such examples exist in this setting, henceforth referred to as the "boundary set setting". See [Cu].

MaIn Result. Let $F$ be a $\sigma$-Z-set in the locally compact separable ANR $Y$. Then $X=Y-F$ satisfies the discrete $n$-cells property for each nonnegative integer $n$ if and only if $X$ satisfies the discrete approximation property.

Received by the editors March 30, 1984 and, in revised form, May 21, 1984.

1980 Mathematics Subject Classification. Primary 57N20.

Key words and phrases. Discrete approximation property, discrete $n$-cells property.

(1985 American Mathematical Society $0002-9939 / 85 \$ 1.00+\$ .25$ per page 
The following Corollary is a trivial consequence of $\mathrm{H}$. Torunczyk's Hilbert space manifold characterization [To2, BBMW] and the Main Result.

Corollary. A $\sigma$-Z-set $B$ in the Hilbert cube manifold $M$ is a boundary set (that is, $M-B$ is a Hilbert space manifold) if and only if $M-B$ satisfies the discrete $n$-cells property for each nonnegative integer $n$.

In the final section, $\S 4$, we include a brief discussion of further consequences that the Main Result holds for Hilbert space manifold characterizations. In particular, in the boundary set setting, Hilbert space manifolds are characterized in terms of their homological structure combined with a minimal amount of general positioning.

Terminology and notation. All spaces are assumed to be separable and metrizable, and maps are continuous functions. If $K$ is an abstract simplicial complex, we use standard abuse of notation and also write $K$ for the standard geometric realization of $K$ and $K^{(n)}$ for both the abstract $n$-skeleton of $K$ and its standard geometric realization. If $f$ is a map of a space $X$ into a space $Y$, we say that $f$ is strongly approximable by maps $g$ with property P provided that for every open cover $\mathscr{U}$ of $Y$ there exists a map $g$ with property $\mathbf{P}$ such that $f$ and $g$ are $\mathscr{U}$-close. $\mathbf{N}$ denotes the collection of positive integers.

2. Discrete properties. A collection of $\mathscr{D}$ of subsets of a space $X$ is discrete in $X$ provided that every point in $X$ has a neighborhood that meets at most one member of $\mathscr{D}$. It is easy to see that if $\mathscr{D}$ is a discrete collection in $X$, then

$$
\overline{\mathscr{D}}=\left\{\mathrm{Cl}_{X} D \mid D \in \mathscr{D}\right\}
$$

also is discrete. H. Torunczyk [To2] has obtained the following topological characterization of the pseudo-interior $s$ of the Hilbert cube. ${ }^{1}$

THEOREM 2.1. A topologically complete separable AR $X$ is homeomorphic to $s$ if and only if every map $f: \oplus_{i=1}^{\infty} I_{i}^{\infty} \rightarrow X$ of the countable free union of Hilbert cubes into $X$ is strongly approximable by maps $g: \bigoplus_{i=1}^{\infty} I_{i}^{\infty} \rightarrow X$ for which the collection $\left\{g\left(I_{i}^{\infty}\right)\right\}_{i=1}^{\infty}$ is discrete.

The above approximation property is known as the discrete approximation property. If we replace the countable free union of Hilbert cubes by the countable free union of $n$-cells, we obtain a hierarchy of discrete cells properties:

DEFINITION 2.2. A metric space $X$ satisfies the discrete n-cells property for $n \in \mathbf{N} \cup\{0\} \cup\{\infty\}$ (discrete $\infty$-cells property = discrete approximation property) if for each map $f: \bigoplus_{i=1}^{\infty} I_{i}^{n} \rightarrow X$ and each open cover $\mathscr{U}$ of $X$ there exists a map $g$ : $\oplus_{i=1}^{\infty} I_{i}^{n} \rightarrow X$ such that $f$ and $g$ are $\mathscr{U}$-close and $\left\{g\left(I_{i}^{n}\right)\right\}_{i=1}^{\infty}$ is discrete.

THEOREM 2.3. A topologically complete separable ANR X satisfies the discrete $n$-cells property if and only if every map from any topologically complete separable $n$-dimensional space into $X$ is strongly approximable by closed embeddings.

This theorem is due to $\mathrm{H}$. Torunczyk and all the ingredients for its proof appear in [To2]. A complete proof appears in [Bo2].

\footnotetext{
${ }^{1}$ There is a mistake in Torunczyk's proof. A correct proof appears in [BBMW].
} 
The following lemma is used in the proof of the Main Result of $\S 3$. Though this result never explicitly appears in [To2], all the ingredients for its proof appear there.

LEMMA 2.4. Let $X$ be a topologically complete separable ANR that satisfies the discrete $n$-cells property for each nonnegative integer $n$, and let $\left\{K_{i}\right\}_{i=1}^{\infty}$ be a sequence of locally finite countable complexes. Then for every map $\nu: \oplus_{i=1}^{\infty} K_{i} \rightarrow X$ and every open cover $\mathscr{U}$ of $X$ there exists a map $\nu^{\prime}: \bigoplus_{i=1}^{\infty} K_{i} \rightarrow X$ such that $\nu^{\prime}$ is $\mathscr{U}$-close to $\nu$ and, for each nonnegative integer $n$, the collection $\left\{\nu^{\prime}\left(K_{i}^{(n)}\right)\right\}_{i=1}^{\infty}$ is discrete.

Proof. For each nonnegative integer $n$ let

$$
V_{n}=\left\{f: \bigoplus_{i=1}^{\infty} K_{i} \rightarrow X \mid\left\{f\left(K_{i}^{(n)}\right)\right\}_{i=1}^{\infty} \text { is discrete }\right\} .
$$

Then each $V_{n}$ is an open subset of the function space $C\left(\oplus_{i=1}^{\infty} K_{i}, X\right)$ with the limitation topology (see [To2, §1]). Using the facts that $\dot{X}$ satisfies the discrete $n$-cells property and $X$ is a topologically complete ANR, it is a straightforward consequence of Theorem 2.3 that $V_{n}$ is dense in $C\left(\oplus_{i=1}^{\infty} K_{i}, X\right)$. Since $X$ is topologically complete, Lemma 1.1 of [To2] guarantees that $C\left(\oplus_{i=1}^{\infty} K_{i}, X\right)$ is a Baire space and, therefore, $\cap_{n=1}^{\infty} V_{n}$ is dense in $C\left(\oplus_{i=1}^{\infty} K_{i}, X\right)$. This is equivalent to the stated conclusion of the lemma.

3. The main results. Recall that a Z-set $F$ in an ANR $X$ is a closed subset of $X$ such that the identity map on $X$ is strongly approximable by maps into $X-F$. A $\sigma$-Z-set is a countable union of $Z$-sets in $X$. A standard argument shows that if $F$ is a $\sigma$ - $Z$-set in a topologically complete separable ANR $X$, then the identity map on $X$ is strongly approximable by maps into $X-F$.

The Main Result is stated in the introduction, and the remainder of this section is devoted to its proof. Our first task reduces the Main Result to a question concerning the structure of topologically complete separable ANR's. We then show that those ANR's that arise as complements of $\sigma$ - $Z$-sets in locally compact separable ANR's have the desired structure.

LEMMA 3.1. Let $X$ be a topologically complete separable ANR that satisfies the discrete $n$-cells property for each nonnegative integer $n$. Then $X$ satisfies the discrete approximation property if and only if $X$ satisfies the following property:

For every open cover $\mathscr{U}$ of $X$ there exists a countable open cover $\mathscr{V}=\left\{V_{i}\right\}_{i=1}^{\infty}$ of $X$ and a locally finite countable complex $K$ with (*) maps $\mu: X \rightarrow K$ and $\nu: K \rightarrow X$ and positive integers $n(1), n(2), \ldots$ such that $\nu \circ \mu$ is $\mathscr{U}$-close to $\mathrm{id}_{X}$ and, if $\sigma$ is a simplex in $K$ of dimension greater than $n(i)$, then $\nu(\sigma) \cap V_{i}=$ $\varnothing$.

Proof. Suppose $X$ satisfies the discrete approximation property and $\mathscr{U}$ is an open cover of $X$, and let $\mathscr{W}$ be a star refinement of $\mathscr{U}$. Since $X$ is a separable ANR, there exists a locally finite countable complex $K$ and maps $\mu: X \rightarrow K$ and $\nu_{*}: K \rightarrow X$ such that $\nu_{*} \circ \mu$ is $\mathscr{W}$-close to $\mathrm{id}_{X}$. Since $X$ satisfies the discrete approximation property, 
Theorem 2.3 with $n=\infty$ guarantees that there exists a closed embedding $\nu: K \rightarrow X$ that is $\mathscr{W}$-close to $\nu_{*}$. Notice that $\nu \circ \mu$ is then $\mathscr{U}$-close to id ${ }_{X}$. Write $K$ as an increasing union of compact subcomplexes of $K$ as follows: $K=\cup_{i=1}^{\infty} K_{i}$, where each $K_{i}$ is a finite collection of simplices in $K$ and $K_{i} \subset$ Int $K_{i+1}$. Let $n(i)$ denote the dimension of the highest-dimensional simplices in $K_{i+1}$. Since $\nu$ is a closed embedding of $K$ into $X$, for each $i$ we can find an open set $V_{i}$ in $X$ such that $\nu\left(K_{i}\right) \subset V_{i}$ and $V_{i} \cap\left(\nu\left(K-\right.\right.$ Int $\left.\left.K_{i+1}\right)\right)=\varnothing$. Since $\nu(K)$ is closed in $X$, we may assume that $V_{1}$ contains $X-\nu(K)$ so that $\mathscr{V}=\left\{V_{i}\right\}_{i=1}^{\infty}$ covers $X$. If $\sigma$ is a simplex in $K$ of dimension greater than $n(i)$, then $\sigma \notin K_{i+1}$, and since $V_{i} \cap\left(\nu\left(K-\right.\right.$ Int $\left.\left.K_{i+1}\right)\right)=\varnothing$, $\nu(\sigma) \cap V_{i}=\varnothing$.

We now prove the reverse implication. Let $\mathscr{U}$ be an open cover of $X$ and let $\mathscr{V}, K, \mu, \nu, n(1), n(2), \ldots$ be as hypothesized in (*). Let $K_{i}=K$ for $i=1,2, \ldots$ and let $\bar{\nu}: \bigoplus_{i=1}^{\infty} K_{i} \rightarrow X$ be defined by $\bar{\nu} \mid K_{i}=\nu$. It suffices to show that there exists a map $\nu^{\prime}: \bigoplus_{i=1}^{\infty} K_{i} \rightarrow X$ that is $\mathscr{U}$-close to $\bar{\nu}$ and for which $\left\{\nu^{\prime}\left(K_{i}\right)\right\}_{i=1}^{\infty}$ forms a discrete family.

Choose an open cover $\mathscr{W}$ of $X$ that refines $\mathscr{U}$ and star refines $\mathscr{V}$, and apply Lemma 2.4 to $\bar{\nu}$ and $\mathscr{W}$ to obtain a map $\nu^{\prime}: \bigoplus_{i=1}^{\infty} K_{i} \rightarrow X$ that is $\mathscr{W}$-close to $\bar{\nu}$ and for which $\left\{\nu^{\prime}\left(K_{i}^{(n)}\right)\right\}_{i=1}^{\infty}$ is discrete for each nonnegative integer $n$. Obviously, $\left\{\nu^{\prime}\left(K_{i}\right)\right\}_{i=1}^{\infty}$ is pairwise disjoint. Let $x \in X$ and suppose there are points $y_{i} \in K_{k(i)}$ such that $\nu^{\prime}\left(y_{i}\right) \rightarrow x$ for some positive integers $k(i)$. Since $\nu^{\prime}$ is $\mathscr{W}$-close to $\bar{\nu}$, there exists $W_{i}, W \in \mathscr{W}$, such that $\left\{\bar{\nu}\left(y_{i}\right), \nu^{\prime}\left(y_{i}\right)\right\} \subset W_{i}$ and $x \in W$. Thus, we may assume that $\left\{\bar{\nu}\left(y_{i}\right)\right\}_{i=1}^{\infty} \subset \operatorname{st}(W, \mathscr{W})$. Since $\mathscr{W}$ star refines $\mathscr{V}$, there exists $V_{p} \in \mathscr{V}$ such that $\left\{\bar{\nu}\left(y_{i}\right)\right\}_{i=1}^{\infty} \subset V_{p}$. By $(*)$, since $\bar{\nu}\left(y_{i}\right)=\nu\left(y_{i}\right), y_{i} \in K_{k(i)}^{(n(p))}$ for each $i$. Since $\left\{\nu^{\prime}\left(K_{i}^{(n(p))}\right)\right\}_{i=1}^{\infty}$ is discrete, there must be a positive integer $k$ such that $k(i)=k$ for all but finitely many $i$. This implies that $\left\{\nu^{\prime}\left(K_{i}\right)\right\}_{i=1}^{\infty}$ is discrete.

We now prove that $X$ satisfies property (*) provided $X$ arises as the complement of a $\sigma-Z$-set in a locally compact separable ANR.

LemMA 3.2. Let $Y$ be a locally compact separable ANR, and let $F$ be a $\sigma$-Z-set in $Y$. Then $X=Y-F$ satisfies $(*)$.

Proof. Let $\mathscr{U}$ be an open cover of $X$ and choose a collection $\mathscr{W}$ of sets open in $Y$ such that $\mathscr{U}=X \cap \mathscr{W}=\{X \cap W \mid W \in \mathscr{W}\}$. Let $Z=\bigcup\{W \mid W \in \mathscr{W}\}$, an open subset of $Y$, and write $Z$ as an increasing union of compacta; that is, $Z=\bigcup_{i=1}^{\infty} Z_{i}$, where each $Z_{i}$ is compact and satisfies $Z_{i} \subset$ Int $Z_{i+1}$. We prove the following claim later.

Claim. There exists a locally finite countable complex $K$, maps $\bar{\mu}: Z \rightarrow K$ and $\bar{\nu}$ : $K \rightarrow Z$, and positive integers $n(1), n(2), \ldots$ such that $\bar{\nu} \circ \bar{\mu}$ is $\mathscr{W}$-close to $\mathrm{id}_{Z}$ and, if $\sigma$ is a simplex in $K$ of dimension greater than $n(i)$, then $\bar{\nu}(\sigma) \cap Z_{i}=\varnothing$.

Since $F$ is a $\sigma$ - $Z$-set in $Y$ and $Z$ is open in $Y, F \cap Z$ is a $\sigma$ - $Z$-set in $Z$ and there is a map $u: Z \rightarrow Z-F=X$ that is $\mathscr{W}$-close to $i_{Z}$ such that $u\left(Z-Z_{i+1}\right) \subset Z-Z_{i}$ for each $i$. Let $\mu=\bar{\mu} \mid X, \nu=u \circ \bar{\nu}, V_{1}=\varnothing, V_{i}=\left(\right.$ Int $\left.Z_{i-1}\right) \cap X$ for $i>1$, and $\mathscr{V}$ $=\left\{V_{i}\right\}_{i=1}^{\infty}$. Since necessarily $X$ is dense in $Z$, it is easy to show that $\nu \circ \mu$ is st $\mathscr{U}$-close to id ${ }_{X}$. If $\sigma$ is a simplex in $K$ of dimension greater than $n(i)$, then $\bar{\nu}(\sigma) \cap Z_{i}=\varnothing$ and, therefore, since $u\left(Z-Z_{i}\right) \subset Z-Z_{i-1}, \nu(\sigma) \cap V_{i}=\varnothing$. Thus, modulo the proof of the Claim, $X$ satisfies $(*)$. 
Proof of the Claim. Let $\mathscr{A}$ be an open refinement of $\mathscr{W}$ such that if $A \in \mathscr{A}$ and $A \cap Z_{i} \neq \varnothing$, then $A \subset Z_{i+1}$. This implies that if $A \cap\left(Z_{i+1}-Z_{i}\right) \neq \varnothing$, then $A \cap Z_{i-1}=\varnothing$. Let $\mathscr{B}$ be a star refinement of $\mathscr{A}$. Since $Z$ is an ANR, there exists an open refinement $\mathscr{C}$ of $\mathscr{B}$ such that partial $\mathscr{C}$-realizations of complexes in $Z$ extend to full $\mathscr{B}$-realizations of complexes in $Z$. Let $\mathscr{D}$ be a countable star finite open refinement of a star refinement of $\mathscr{C}$. Fix a positive integer $i$. Since $Z_{i}$ is compact and $\mathscr{D}$ is star finite, all but finitely many elements of $\mathscr{D}$ miss $Z_{i}$. Let $\mathscr{D}_{i}$ denote the collection of elements of $\mathscr{D}$ that intersect $Z_{i}$ nontrivially, and let $n(i)$ denote the order of $\mathscr{D}_{i+1}$; that is, $n(i)$ is the largest integer for which there are $n(i)+1$ members of $\mathscr{D}_{i+1}$ that intersect nontrivially.

Let $K=\mathscr{N}(\mathscr{D})$, the nerve of $\mathscr{D}$, and let $\bar{\mu}: Z \rightarrow K$ be a standard map for which $\bar{\mu}^{-1}$ (open star of $\left.D\right) \subset D$ for each $D \in \mathscr{D}$. Define $\bar{\nu} \mid K^{(0)}$ by $\bar{\nu}(D)=d$, where $d$ is some prechosen element of $D$ in $\mathscr{D}$. Let $\sigma=\left\langle D_{0}, \ldots, D_{n}\right\rangle \in K$. Then $\bigcap_{i=0}^{n} D_{i} \neq \varnothing$ and, since $\mathscr{D}$ star refines $\mathscr{C}, \bar{\nu}\left(\sigma^{(0)}\right) \subset C$ for some $C$ in $\mathscr{C}$. Thus, $\bar{\nu} \mid K^{(0)}$ is a partial $\mathscr{C}$-realization of $K$ in $Z$ and there exists a full realization $\bar{\nu}: K \rightarrow Z$ such that each $\bar{\nu}(\sigma)$ for $\sigma$ in $K$ is contained in some $B$ in $\mathscr{B}$.

We leave the reader the task of showing that $\bar{\nu} \circ \bar{\mu}$ is $\mathscr{W}$-close to id $z$. Let $\sigma$ be a simplex in $K$ of dimension greater than $n(i)$, say, $\sigma=\left\langle D_{0}, \ldots, D_{n}\right\rangle$, where $n>n(i)$. Since $\bigcap_{k=0}^{n} D_{k} \neq \varnothing$, we may assume without loss of generality that $D_{0}$ is not an element of $\mathscr{D}_{i+1}$; that is, $D_{0} \cap Z_{i+1}=\varnothing$. This follows from the definition of $n(i)$. Thus there exists an integer $k>i+1$ such that $D_{0} \cap\left(Z_{k}-Z_{k-1}\right) \neq \varnothing$. There exists sets $B$ and $B^{\prime}$ in $\mathscr{B}$ such that $\bar{\nu}(\sigma) \subset B$ and $D_{0} \subset B^{\prime}$ and, since $\bar{\nu}\left(D_{0}\right) \in D_{0}$, $B \cap B^{\prime} \neq \varnothing$. Therefore, there exists $A \in \mathscr{A}$ with $\bar{\nu}(\sigma) \cup D_{0} \subset A$. Since $D_{0} \subset A$, $A \cap\left(Z_{k}-Z_{k-1}\right) \neq \varnothing$ and, thus, $A \cap Z_{k-2}=\varnothing$. Since $Z_{i} \subset Z_{k-2}, A \cap Z_{i}=\varnothing$ and, therefore, $\bar{\nu}(\sigma) \cap Z_{i}=\varnothing$. This complete the proof of the Claim.

The proof of the Main Result follows directly from Lemmas 3.1 and 3.2.

4. Final remarks. Following standard practice we use the abbreviation $Q$-manifold (resp., $l_{2}$-manifold) to denote Hilbert cube (resp. Hilbert space) manifolds. $R$. Daverman and J. Walsh in [DW] refine H. Torunczyk's $Q$-manifold characterization [To1] by showing that $Q$-manifolds are characterized among locally compact ANR's by combining the disjoint 2-cells property with a disjoint Čech carriers property; roughly, the latter property is that, at the level of Čech homology, homology elements can be made disjoint. A locally compact ANR $X$ is said to be a $Q$-manifold factor provided there is a finite-dimensional space $Y$ such that $X \times Y$ is a $Q$-manifold. The Daverman-Walsh characterization is important because it provides a characterization of $Q$-manifold factors: a locally compact ANR $X$ is a $Q$-manifold factor if and only if $X$ satisfies the disjoint Čech carriers property.

In [Bo1] the author attempts a characterization of $l_{2}$-manifolds in the spirit of Daverman and Walsh. There the following Theorem is proved.

THEOREM. Let $X$ be a topologically complete separable ANR. If $X$ satisfies the discrete 2-cells property and the discrete carriers property, then $X$ satisfies the discrete $n$-cells property for each nonnegative integer $n$.

The discrete carriers property roughly states that, at the level of singular homology, any sequence of homology elements can be made discrete. 
The Main Result coupled with this Theorem and H. Torunczyk's $l_{2}$-manifold characterization show that $l_{2}$-mainfolds that appear in the boundary set setting are precisely those complements of $\sigma$-Z-sets in locally compact separable ANR's that satisfy the discrete carriers property and the discrete 2-cells property. The author has recently proved that certain $l_{2}$-manifold factors that appear in the boundary set setting are those complements of $\sigma$ - $Z$-sets in locally compact separable ANR's that satisfy the discrete carriers property.

It was hoped that this characterization of those $l_{2}$-manifolds that arise in the boundary set setting would provide a homological characterization of $l_{2}$-manifolds in general; however, [BBMW] contains a description of an example of a topologically complete separable ANR $X$ that satisfies the discrete carriers property and the discrete $n$-cells property for each nonnegative integer $n$, yet fails to be an $l_{2}$-manifold. The fact that $X$ is not an $l_{2}$-manifold is detected by observing that $X$ does not satisfy the discrete approximation property. See [BBMW, Bo1, DW].

\section{REFERENCES}

[BBMW] M. Bestvina, P. Bowers, J. Mogilski and J. Walsh, Characterization of Hilbert space manifolds revisited, preprint.

[Bo1] P. L. Bowers, Applications of general position properties of dendrites to Hilbert space topologv, Ph.D. Diss., Univ. of Tennessee, 1983.

[Bo2] __ Dense embeddings of sigma-compact, nowhere locally compact metric spaces, preprint.

[Cu] D. W. Curtis, Boundary sets in the Hilbert cube, Topology and Appl. (to appear).

[DW] R. J. Daverman and J. J. Walsh, Čech homology characterizations of infinite dimensional manifolds, Amer. J. Math. 103 (1981), 411-435.

[To1] H. Torunczyk, On CE-images of the Hilbert cube and characterization of $Q$-manifolds, Fund. Math. 106 (1980), 31-40.

[To2] __ Characterizing Hilbert space topology, Fund. Math 111 (1981), 247-262.

Department of Mathematics, University of Tennessee, Knoxville, Tennessee 37996

Current address: Department of Mathematics, Florida State University, Tallahassee, Florida 32306 